\title{
Dynamics of Parasympathetic Activity in Violent Incarcerated Offenders Before, During, and in Recovery From An Emotional Inhibition Task
}

Julie Palix ( $\nabla$ julie.palix@chuv.ch )

Research Unit of Legal Psychiatry and Psychology, University Hospital of Lausanne,

Steven M. Gillespie

Department of Primary Care and Mental Health, University of Liverpool, Liverpool

Milena Abbiati

Research Unit of Legal Psychiatry and Psychology, University Hospital of Lausanne,

Ahmad Abu-Akel

Institute of Psychology, University of Lausanne, Lausanne

\section{Research Article}

Keywords: Violence, parasympathetic activity, heart rate variability, resting state, recovery, go/no-go task

Posted Date: December 29th, 2021

DOI: https://doi.org/10.21203/rs.3.rs-1188153/v1

License: (a) (i) This work is licensed under a Creative Commons Attribution 4.0 International License.

Read Full License 


\section{Abstract}

Dynamics of the autonomic nervous system (ANS) are hypothesized to play a role in the emergence of interpersonal violence. In the present study, we examined continuous activities of the inhibitory parasympathetic pathway of the ANS through the root mean square of successive differences between normal heartbeats (RMSSD) in 22 male offenders who committed interpersonal violence and 24 matched controls from the general population across three successive phases: resting baseline, while performing an emotional Go/No-Go task, and post-task recovery. Results showed that across the three phases, the offender group presented lower RMSSD at baseline $\left(p_{F D R}=.003\right.$; Cohen's $\left.d=-1.11\right)$, but similar levels during the task, attributed to a significant increase in their RMSSD level ( $p_{F D R}=.027$, Cohen's $\left.d=-1.26\right)$. During recovery, no distinction between the two groups was found, but although both groups showed signs of recovering toward baseline values. These findings suggest that violent incarcerated offenders can flexibly engage parasympathetic resources to meet environmental challenges. This underscores the necessity of considering parasympathetic dynamics and its respective mobilization/flexibility to better understand ANS profiles underlying interpersonal violence and designed more tailored intervention.

\section{Introduction}

Several studies of interpersonal violence have linked Autonomic Nervous System (ANS) activity at rest and in response to threat or provocation with relationally aggressive behaviors (for reviews see (Branje \& Koot, 2018; Murray-Close, Breslend, \& Holterman, 2018). The ANS consists of two antagonistic pathways: the parasympathetic and sympathetic systems (PNS and SNS, respectively). The PNS acts as a brake on the intrinsic rate of the heart to respond rapidly to changing metabolic demands, whether from emotional arousal or from a simple posture change (Berntson et al., 1994; Larsen, Schneiderman, \& Pasin, 1986; Roos et al., 2017; Thayer, Hansen, Saus-Rose, \& Johnsen, 2009). Its involvement is fast to allow a rapid inhibition of the predominant alarm-type, flight-or-fight, SNS activity (Geisler, Kubiak, Siewert, \& Weber, 2013; McCraty \& Shaffer, 2015). Thus, heightened PNS activation promotes restorative functions and allows social adaptability and psychological flexibility, while weakened PNS activity is indicative of psychiatric conditions or poor self-regulatory control (Berntson \& Cacioppo, 2004; Camm et al., 1996; Thayer et al., 2009). Accordingly, fluctuations in PNS activity - typically measured via continuous recordings of the successive inter-beat intervals of the heart, also called heart rate variability (HRV) (Camm et al., 1996; Nunan, Sandercock, \& Brodie, 2010; Shaffer \& Ginsberg, 2017)-is of particular interest to understand the mechanisms underpinning behavioral and emotional regulation (Roos et al., 2017; Thayer et al., 2009), and putatively, in understanding the loss of control or panic that could occur in episodes of violence (Haller et al., 2014; McQuade et al., 2019; Palix, Akselrod, Cungi, Giuliani, \& Favrod, 2017; Umhau et al., 2002).

HRV research concerned with the psychophysiological mechanisms underlying violent behaviour has mainly focused on the resting state, measured over a 5-minute period of calm (Kassing, Lochman, \& Glenn, 2018; Palix et al., 2021; Williams, Steptoe, Chambers, \& Kooner, 2011). While this body of research has consistently shown an association between attenuated levels of PNS at rest (low HRV) and high 
levels of traits hostility, anger, aggression, and weaker fear extinction (Demaree \& Everhart, 2004; Haller et al., 2014; Pappens et al., 2014; Puhalla, Kulper, Fahlgren, \& McCloskey, 2020), studying HRV beyond the at-rest phase might provide important new insights about violent behavior. For example, a recent study highlighted that the inclusion of HRV during a negative mood induction task improved the prediction of violent recidivism in young adults (de Ruigh et al., 2020).

The importance of characterizing the dynamics of HRV (i.e., during Rest, Reactivity, and Recovery) is further highlighted by a recent theoretical account, which advocates for the need to examine HRV through these different phases, in order to more fully understand how efficiently self-regulatory resources are mobilized and used (Laborde, Mosley, \& Mertgen, 2018). In their introduction of The Vagal Tank Theory, Laborde et al. (2018) use the metaphor of the tank to represent the self-regulatory resources at one's disposal during Rest, which are depleted in Reactivity to an event (such as in completing a task) and then replenished to baseline levels post-event during Recovery, in order to face any other upcoming demanding event. According to this theory, the magnitude of the decrease between the baseline and the active phase would correspond to the metabolic demand required by the task, and is expected to be small in, for example, the case of sustained attention (Tracy \& Giummarra, 2017) or simple reaction tasks (Berntson et al., 1994), and near full during a fight or flight response (Beauchaine, Gatzke-Kopp, \& Mead, 2007; Porges, 2007). However, some increase in vagal control (increased HRV) from rest to task-related mobilization cannot be ruled out, especially in tasks requiring emotional resources (Park, Vasey, Van Bavel, \& Thayer, 2014) or empathic skills (Di Palma et al., 2019; Sassenrath, Barthelmäs, Saur, \& Keller, 2021).

Little is known about HRV reactivity (from baseline to task), and recovery post-event in violent offenders. In this study, we examine the PNS dynamics in individuals who have already committed a serious act of interpersonal violence, and how this might inform strategies for the development of self-regulation abilities in this population. Specifically, the current study examines changes in HRV when presented with a cognitively demanding task, how these changes are associated with differences in behavioral performance, and how these resources recover post-event. For the task phase, we chose the emotional Go/No-Go task, which requires individuals to discriminate stimuli of different emotional valences as well as to inhibit a prepotent response (Murphy et al., 1999). Since it involves processes that have been linked to changes in different frequency bands of HRV (Park et al., 2014; Roos et al., 2017), this task can highlight differences in autonomic flexibility when challenged. Accordingly, the current study examines the functional dynamics of HRV (PNS activity) during alternating phases of rest, reactivity to an emotional Go/No-Go task, and recovery in violent offenders compared to matched controls. Based on results from previous studies (Murray-Close, Holterman, Breslend, \& Sullivan, 2017; Patrick, 2008; Puhalla et al., 2020; Puhalla \& McCloskey, 2020), and drawing on the predictions of the Vagal Tank Theory (Laborde et al., 2018), we predicted that the offender group would show low levels of resting HRV, indicative of poorer self-regulatory capacities. Specifically, we predict that the offender group will show a) reduced HRV at rest compared to controls, and b) limited reactivity from the baseline to task, and c) less efficient recovery during the post-task phase. Moreover, because of lower expected HRV, and associated problems in response inhibition and self-regulation, we expect the offender group to underperform the control group in the Go/No-Go task. For the control group, HRV at rest is expected to attenuate during 
activity while performing the task to accommodate the mental effort needed (Berntson et al., 1994; Delaney \& Brodie, 2000), and a restoration of baseline levels during the recovery phase.

\section{Material And Methods Participants}

The study sample consisted of 22 incarcerated male offenders (mean age $=39.27$ (SD $=12.12$ ) years, range 24 to 72 ) and 24 male non-offender controls from the general population (mean age $=32.46(\mathrm{SD}=$ 12.47) years, range 19 to 58), matched in terms of age, body mass index and education (all $p s>.05$ ). The average time spent in custody by offenders at the time of the experiment was 5.95 (SD $=4.68$ ) years. The mean length of their prison sentence was $11.88(S D=7.03)$ years. Over half of the participants in the offender group had been convicted of homicide $(54 \%, 12 / 22)$, followed by homicide attempt $(32 \%, 7 / 22)$ and other forms of violence $(14 \%, 3 / 22)$. Time between crime and testing was about 7.6 years $(S D=7.3)$. Most of them knew their victim $(68 \%, 15 / 22)$, and acted without premeditation $(68 \%, 15 / 22)$. According to psychiatric or medical referent records, $45 \%$ (10/22) of the sample had previously been diagnosed with personality disorder, $23 \%(5 / 22)$ with psychosis, and $32 \%(7 / 22)$ without any major psychiatric problems. Over half of the sample $(55 \%, 12 / 22)$ were Swiss nationals, $36 \%(8 / 22)$ from the EU, and $9 \%(2 / 22)$ outside the EU.

Inclusion criteria. Offenders were all male, French speakers, at least 18 years old, and had committed one or more acts of interpersonal violence. We excluded participants who had committed other types of violence (e.g., sexual or against property), were awaiting trial, or could not be tested due to health problems. The testing took place in a dimly lit, quiet room, alone with the experimenter, with a prison officer outside the door following prison authority's protocol.

We recruited the controls by approaching participants during the mandatory recruitment days of the Swiss army and through public announcements. We tested participants with the same protocol as the offender group, in a dimly lit, quiet room, alone with the experimenter. $75 \%(18 / 24)$ were Swiss nationals, and $25 \%(6 / 24)$ from the EU. The testing took place either in a room at the army trainee garrisons $(6 / 24$ participants), a room in the university hospital (13/24 participants), or at their domicile (5/24 participants). Participation was voluntary. Participants received $40 \mathrm{CHF}$ compensation, except for the young army recruits, at the request of the Swiss army administration. As confirmed in the personal interview, none of the controls reported a criminal record for a violent offence against another person.

\section{Procedure}

We installed an electrocardiogram (ECG) belt comfortably positioned in a closed, dimly lit room. Following a short stabilization period, the baseline ECG (5 min following the guidelines of the Task Force (Camm et al., 1996)) was obtained first with eyes-opened. The ECG recording continued while participants performed the task (4 min on average), and then for an additional $5 \mathrm{~min}$, at rest, with eyesopened. The whole session lasted about an hour including the instructions period as well as the 
installation and removal of the belt. All participants, offenders as controls, provided their signed informed consent before participation. The study was carried out in accordance with the World Medical Association declarations of Helsinki, and approved by the Ethics Committee of the University of Lausanne (CER-VD 58/14).

HRV acquisition and analysis. We acquired HRV using a wireless ECG (Equivital system, Cambridge, UK), and processed with the Kubios HRV Premium Software, Version 3.0.2 (Kubios Oy 2016-2019, https://www.kubios.com, Kuopio, Finland) to transform the inter-beat variability into PNS activity estimate. HRV values are measured and reported using the time domain RMSSD (Root Mean Square of Successive Differences, in milliseconds) (Camm et al., 1996; Zygmunt \& Stanczyk, 2010), with higher values representing stronger parasympathetic activity. Norms for RMSSD in the general population are reported to average $42 \mathrm{~ms} \pm 15$ (Nunan et al., 2010). The RMSSD values were computed over three phases: (1) baseline, (2) task, and (3) recovery for both samples, independently.

Task. We employed a shortened version of the emotional Go/No-Go task (Drevets \& Raichle, 1998), to accommodate time constraints for testing in the prison environment. The task was administered using $\mathrm{E}-$ Prime version 2.0 (Psychology Software Tools, Inc., Pittsburgh, PA), and consisted of two blocks of 60 trials each: $42(75 \%)$ Go trials, and $12(25 \%)$ No-Go trials. Each trial consisted of the presentation of a black-and-white picture of an adult's face expressing either happiness or fear, mouth-opened, for $500 \mathrm{ms,}$ separated by $1000 \mathrm{~ms}$ inter-stimulus intervals. Faces were selected from a set of 12 models ( 6 females and 6 males) from the NimStim dataset (Tottenham et al., 2009). In the first block, participants were asked to press Go when they saw a happy face and to withhold responses to a fearful face. The reverse was the case in the second block. Performance in each block and overall was estimated using the sensitivity index $d$ prime (ability to discriminate between happy and fear faces), obtained by subtracting the $z$-transformed false alarm rate from the $z$-transformed hit rate.

Statistical analysis. Differences between the violent offender and control groups in task performance and ECG indices were tested using independent-samples $t$-tests. The dynamics of RMSSD responsiveness were investigated in one repeated measures analysis of variance (ANOVA), in which we examined the effect of group (violent offenders vs. controls), phase (baseline, task, recovery) and their interaction on the standardized values of RMSSD. All analyses were reported using false discovery rate correction at $p_{\mathrm{fdr}}$ $<0.05$ to control the overall false positive rate. Effect sizes are reported in terms of Cohen's $d$ and partial eta squared $\left(\eta_{p}^{2}\right)$.

\section{Results}

\section{Task performance}

Independent samples $t$-tests revealed no significant differences between the groups in performance on the Go Fearful/No-Go Happy face trials ( $d$ prime MD(se) $=-0.51(0.26), t_{\mathrm{df}=44}=-1.94, p_{\mathrm{FDR}}=0.087$, Cohen's $d=-0.59)$, the Go Happy/No-Go Fearful face trials $\left(d\right.$ prime MD(se) $=-0.46(0.31), t_{\mathrm{df}=44}=-1.46, p_{\mathrm{FDR}}=$ 
0.152 , Cohen's $d=-0.44)$, or the task overall ( $d$ prime $\mathrm{MD}(\mathrm{se})=0.50(0.25), t_{\mathrm{df}=44}=-2.00, p_{\mathrm{FDR}}=0.087$, Cohen's $d=-0.60$ ).

\section{Preliminary Analyses}

Table 1 shows the results of independent samples $t$-tests comparing RMSSD in the offender and control groups at the three experimental phases. The offender group presented significantly lower mean RMSSD during baseline compared to controls. The heart rate values are reported as a general reference and did not differ between groups and phases.

Table 1

Mean and standard deviation (SD) of the RMSSD in the offender and control groups

\begin{tabular}{|c|c|c|c|c|c|c|c|c|}
\hline \multirow[t]{2}{*}{ Variable } & \multicolumn{2}{|c|}{ Offenders } & \multicolumn{2}{|c|}{ Controls } & \multirow[t]{2}{*}{$t$} & \multirow[t]{2}{*}{$p$} & \multirow[t]{2}{*}{$p_{F D R}{ }^{3}$} & \multirow[t]{2}{*}{$d$} \\
\hline & Mean & SD & Mean & SD & & & & \\
\hline $\begin{array}{l}\mathrm{RMSSD}^{1}\left(\mathrm{~ms}^{2}\right) \text { at } \\
\text { baseline/rest }\end{array}$ & 25.64 & 12.63 & 41.29 & 19.81 & -3.16 & 0.003 & 0.023 & -0.94 \\
\hline RMSSD (ms) during task & 51.14 & 42.44 & 57.02 & 51.69 & -0.42 & 0.677 & 0.710 & -0.12 \\
\hline RMSSD (ms) during recovery & 35.70 & 24.78 & 45.34 & 21.15 & -1.42 & 0.162 & 0.365 & -0.42 \\
\hline $\begin{array}{l}\text { Heart Rate }\left(\mathrm{bpm}^{4}\right) \text { at } \\
\text { baseline/rest }\end{array}$ & 78.67 & 13.24 & 74.33 & 10.27 & 1.25 & 0.218 & 0.392 & 0.37 \\
\hline Heart Rate (bpm) during task & 75.35 & 11.99 & 72.51 & 8.43 & 0.94 & 0.355 & 0.456 & 0.27 \\
\hline $\begin{array}{l}\text { Heart Rate (bpm) during } \\
\text { recovery }\end{array}$ & 74.28 & 11.59 & 71.04 & 8.94 & 1.07 & 0.293 & 0.440 & 0.31 \\
\hline \multicolumn{9}{|c|}{${ }^{1}$ RMSSD $=$ Root Mean Square of the Successive beat-to-beat Differences } \\
\hline \multicolumn{9}{|l|}{$2 \mathrm{~ms}=$ millisecond } \\
\hline \multicolumn{9}{|c|}{${ }^{3} \mathrm{FDR}=$ False Discovery Rate $(q=0.05)$ to correct for multiple comparisons. } \\
\hline${ }^{4} \mathrm{bpm}=$ beats per minute & & & & & & & & \\
\hline
\end{tabular}

\section{RMSSD responsiveness and recovery}

We observed a significant group by phase interaction (Figure $1 ; F(2,88)=5.68, p=0.005, \eta_{p}^{2}=0.114$ ). Mean RMSSD values of the offender group were significantly lower than the control group at baseline $\left(\mathrm{MD}(\mathrm{se})=-0.91(0.25), t_{\mathrm{df}=44}=3.69, p_{F D R}=0.003\right.$, Cohen's $\left.d=-1.11\right)$. There were no significant differences between the groups during either the task $\left(\mathrm{MD}(\mathrm{se})=-0.15(0.25), t_{\mathrm{df}=44}=-0.58, p_{F D R}=0.562\right.$, Cohen's $d=$ $-0.18)$ or recovery $\left(\mathrm{MD}(\mathrm{se})=-0.45(0.25), t_{\mathrm{df}=44}=-1.68, p_{F D R}=0.150\right.$, Cohen's $\left.d=-0.51\right)$ phases. 
Furthermore, while the RMSSD dynamics across the three phases appear to follow curvilinear trends, the tests of within-subject contrasts indicated that these were non-significant in either group (Offenders: $F(1,21)=4.04, p=0.058, \eta_{p}{ }^{2}=0.161$; Controls: $\left.F(1,21)=2.01, p=0.170, \eta_{p}{ }^{2}=0.080\right)$. The linear contrasts were also non-significant ( $p s>0.05$ ). Moreover, pairwise comparisons revealed a significant increase in parasympathetic activity among the offenders during the task relative to baseline $(\mathrm{MD}(\mathrm{se})=-0.38(0.13)$, $t_{\mathrm{df}=21}=-2.89, p_{F D R}=0.027$, Cohen's $\left.d=-1.26\right)$. All other comparisons were non-significant $\left(p s_{F D R}>0.05\right)$.

\section{Discussion}

This study aimed to examine the dynamics of the parasympathetic activity in perpetrators of interpersonal violence before, during, and after an acute laboratory task was performed. As evident by their baseline levels of PNS activity, our findings suggest that offenders' ANS activity is distinguishable from that of controls. This confirms our first hypothesis, and is consistent with earlier work examining physiological correlates of hostility and aggression (Kassing et al., 2018; Puhalla et al., 2020; Sloan et al., 1994). This distinction is supported by the observation that only four offenders out of the 22 tested obtained RMSSD values above $35 \mathrm{~ms}$ at rest, i.e. within $1 / 2$ standard deviation of the expected standard mean of 42ms (Nunan et al., 2010). However, our findings do not support our hypothesis that the offender group would be characterized by low parasympathetic responsivity in the task and recovery phases. Contrary to expectations, we observed that their relatively low levels of resting parasympathetic activity rose to levels that were similar to those of the controls during the task, and that their behavioral performance on the task was also equivalent to that of controls. During the recovery phases, while we observed no statistical differences between the groups, or within group differences compared to their respective baselines, there was a tendency for both groups to revert to baseline levels.

From a physiological perspective, we can assume that this low PNS activity at rest could be related to a weakness in the inhibitory functions of the ANS, leading to a persistent hyper aroused state (Haller et al., 2014; Puhalla et al., 2020). However, the finding of relative increases in PNS activity in the offender group while performing the Go/NoGo inhibition task-to a level similar to controls-is suggestive of flexible use of self-regulatory functions (Holzman \& Bridgett, 2017). This leads us to propose that having low levels of PNS activity at rest in the offender group is not indicative of an overall functional impairment in inhibitory control systems per se.

Instead, the offenders were able to mobilize their autonomic resources in a similar way to the controls during the emotional response inhibition task, during which no statistically significant differences in task performance were discerned. This PNS mobilization has implications for understanding mechanisms underlying interpersonal violence and forensic interventions. First, it demonstrates that despite exhibiting low resting HRV -a marker of a tendency towards violence-, offender participants were nonetheless able to respond with autonomic flexibility and engage self-regulatory systems. This encourages proposals advocating for the inclusion of HRV biofeedback training for people with a history of violence (Gillespie, Garofalo, \& Velotti, 2018; Gillespie, Mitchell, Fisher, \& Beech, 2012). The use of HRV biofeedback training should help to increase HRV, and support offenders to exercise self-regulation outside of situations that 
place cognitive inhibitory demands. Our results further suggest that to be comprehensive, the benefits must be ideally assessed at rest, as well as during activity and recovery. Second, this pattern of dynamics in offenders is also consistent with the suggestion that aggressive and antisocial people can provoke a sense of control that might be necessary when engaging in certain antisocial behaviors, including violent assaults (Gillespie, Brzozowski, \& Mitchell, 2018; Thomson, Gillespie, Centifanti, 2020). Accordingly, we hypothesize that while some interpersonal violence might be precipitated by heightened sympathetic, impulsive tendencies, self-regulatory systems might nonetheless be recruited while performing the act of violence.

\section{Declarations}

\section{Acknowledgments}

We would like to thank the prison administration of the canton of Vaud and the Swiss army for allowing data acquisition. We are very grateful to the participants for giving us their time and trust. Finally, we thank Christine Mohr, Elise Dan-Glauser and Valérie Moulin for their constructive discussions regarding the data acquisition and preliminary results.

\section{Financial support}

We thank the Institute of Legal Psychiatry of the CHUV and the Institute of Psychology of the UNIL for their financial support to conduct this study.

JP, $\mathrm{PhD}$, is a Research Project Manager. Her research is focused on the study of violent behavior and the substantial contribution that psychophysiology can provide in this field. Her contribution was to design the project, analyze the physiological data, interpret the results and write the article.

SMG, PhD, is a Lecturer in Clinical Psychology. His research seeks to understand how differences in socio-affective functioning, and functioning of the autonomic nervous system, can contribute to aggressive and antisocial behavior, including interpersonal violence and sexual abuse. His contribution was to discuss the results and write the article.

MA, PhD, is an Associate Psychologist and Project Manager. Her research interests include risk assessment, coping strategies, implement, and evaluation of interventions. Her contribution was to process the behavioral data.

AAA, PhD, is a research fellow in Psychology. A focus of his research seeks to understand cognitive and biological mechanisms underlying violent and aggressive behavior in clinical and non-clinical populations. His contribution was to process statistical analyses, interpret the results and write the article.

\section{Disclosure Statement}

All authors declare to have no potential or actual conflicts concerning this study. 


\section{References}

1. Beauchaine, T. P., Gatzke-Kopp, L., \& Mead, H. K. (2007). Polyvagal theory and developmental psychopathology: Emotion dysregulation and conduct problems from preschool to adolescence. Biological psychology, 74(2), 174-184.

2. Berntson, G. G., \& Cacioppo, J. T. (2004). Heart rate variability: Stress and psychiatric conditions. Dynamic electrocardiography, 41(2), 57-64.

3. Berntson, G. G., Cacioppo, J. T., Binkley, P. F., Uchino, B. N., Quigley, K. S., \& Fieldstone, A. (1994). Autonomic cardiac control. III. Psychological stress and cardiac response in autonomic space as revealed by pharmacological blockades. Psychophysiology, 31(6), 599-608.

4. Branje, S., \& Koot, H. M. (2018). Psychophysiology of aggression. Handbook of child and adolescent aggression, 84-106.

5. Camm, A. J., Malik, M., Bigger, J. T., Breithardt, G., Cerutti, S., Cohen, R., . . Kleiger, R. (1996). Heart rate variability: standards of measurement, physiological interpretation and clinical use. Task Force of the European Society of Cardiology and the North American Society of Pacing and Electrophysiology.

6. de Ruigh, E. L., Kleeven, A. T. H., Jansen, L. M., de Vries Robbé, M., Vermeiren, R. R., Mulder, E. A., . . . Popma, A. (2020). Predicting youth reoffending after incarceration: added value of protective factors and heart rate variability. The Journal of Forensic Psychiatry \& Psychology, 1-32.

7. Delaney, J., \& Brodie, D. (2000). Effects of short-term psychological stress on the time and frequency domains of heart-rate variability. Perceptual and motor skills, 91(2), 515-524.

8. Demaree, H. A., \& Everhart, D. E. (2004). Healthy high-hostiles: Reduced parasympathetic activity and decreased sympathovagal flexibility during negative emotional processing. Personality and Individual Differences, 36(2), 457-469.

9. Di Palma, M., Arcangeli, E., Lattanzi, D., Gabbiadini, A., Gallucci, M., Cuppini, R., . . Berlingeri, M. (2019). Heart Rate Variability reveals the fight between racially biased and politically correct behaviour. Scientific reports, 9(1), 1-13.

10. Drevets, W. C., \& Raichle, M. E. (1998). Reciprocal suppression of regional cerebral blood flow during emotional versus higher cognitive processes: Implications for interactions between emotion and cognition. Cognition and emotion.

11. Geisler, F. C., Kubiak, T., Siewert, K., \& Weber, H. (2013). Cardiac vagal tone is associated with social engagement and self-regulation. Biological psychology, 93(2), 279-286.

12. Gillespie, S. M., Brzozowski, A., \& Mitchell, I. J. (2018). Self-regulation and aggressive antisocial behaviour: Insights from amygdala-prefrontal and heart-brain interactions. Psychology, Crime \& Law, 24(3), 243-257.

13. Gillespie, S. M., Garofalo, C., \& Velotti, P. (2018). Emotion regulation, mindfulness, and alexithymia: Specific or general impairments in sexual, violent, and homicide offenders? Journal of Criminal Justice, 58, 56-66. 
14. Gillespie, S. M., Mitchell, I. J., Fisher, D., \& Beech, A. R. (2012). Treating disturbed emotional regulation in sexual offenders: The potential applications of mindful self-regulation and controlled breathing techniques. Aggression and Violent Behavior, 17(4), 333-343.

15. Haller, J., Raczkevy-Deak, G., Gyimesine, K. P., Szakmary, A., Farkas, I., \& Vegh, J. (2014). Cardiac autonomic functions and the emergence of violence in a highly realistic model of social conflict in humans. Frontiers in behavioral neuroscience, 8, 364.

16. Holzman, J. B., \& Bridgett, D. J. (2017). Heart rate variability indices as bio-markers of top-down selfregulatory mechanisms: A meta-analytic review. Neuroscience \& biobehavioral reviews, 74, 233-255.

17. Kassing, F., Lochman, J. E., \& Glenn, A. L. (2018). Autonomic functioning in reactive versus proactive aggression: the influential role of inconsistent parenting. Aggressive behavior, 44(5), 524-536.

18. Laborde, S., Mosley, E., \& Mertgen, A. (2018). Vagal tank theory: the three rs of cardiac vagal control functioning-resting, reactivity, and recovery. Frontiers in neuroscience, 12, 458.

19. Larsen, P., Schneiderman, N., \& Pasin, R. D. (1986). Physiological bases of cardiovascular psychophysiology. Psychophysiology: Systems, processes, and applications, 122-165.

20. McCraty, R., \& Shaffer, F. J. (2015). Heart rate variability: new perspectives on physiological mechanisms, assessment of self-regulatory capacity, and health risk. Global advances in health and medecine, 4(1), 46-61.

21. McQuade, J. D., Murray-Close, D., Breslend, N. L., Balda, K. E., Kim, M. M., \& Marsh, N. P. (2019). Emotional underarousal and overarousal and engagement in relational aggression: Interactions between relational victimization, physiological reactivity, and emotional sensitivity. Journal of abnormal child psychology, 47(10), 1663-1676.

22. Murphy, F., Sahakian, B., Rubinsztein, J., Michael, A., Rogers, R., Robbins, T., \& Paykel, E. (1999). Emotional bias and inhibitory control processes in mania and depression. Psychological medicine, 29(6), 1307-1321.

23. Murray-Close, D., Breslend, N. L., \& Holterman, L. A. (2018). Psychophysiology indicators of relational aggression. In C. Ostrov (Ed.), The development of relational aggression (pp. 127-151): Oxford University Press.

24. Murray-Close, D., Holterman, L. A., Breslend, N. L., \& Sullivan, A. (2017). Psychophysiology of proactive and reactive relational aggression. Biological psychology, 130, 77-85.

25. Nunan, D., Sandercock, G. R., \& Brodie, D. A. (2010). A quantitative systematic review of normal values for short-term heart rate variability in healthy adults. Pacing clinical electrophysiology, 33(11), 1407-1417.

26. Palix, J., Abu-Akel, A., Moulin, V., Abbiati, M., Gasser, J., Hasler, C., . . Dan-Glauser, E. (2021). The utility of physiological measures in assessing the empathic skills of incarcerated violent offenders. International journal of offender therapy and comparative criminology, 0306624X21994056.

27. Palix, J., Akselrod, M., Cungi, C., Giuliani, F., \& Favrod, J. (2017). Changes in heart rate variability recorded in natural situation with t-shirt integrated sensors and level of observed behavioral 
excitation: a pilot study of patients with intellectual disabilities and psychiatric disorders. Frontiers in psychiatry, 8, 4.

28. Pappens, M., Schroijen, M., Sütterlin, S., Smets, E., Van den Bergh, O., Thayer, J. F., \& Van Diest, I. (2014). Resting heart rate variability predicts safety learning and fear extinction in an interoceptive fear conditioning paradigm. PloS one, 9(9), e105054.

29. Park, G., Vasey, M. W., Van Bavel, J. J., \& Thayer, J. F. (2014). When tonic cardiac vagal tone predicts changes in phasic vagal tone: The role of fear and perceptual load. Psychophysiology, 51(5), 419426.

30. Patrick, C. J. (2008). Psychophysiological correlates of aggression and violence: an integrative review. Philosophical Transactions of the Royal Society B: Biological Sciences, 363(1503), 25432555.

31. Porges, S. W. (2007). The polyvagal perspective. Biological psychology, 74(2), 116-143.

32. Puhalla, A. A., Kulper, D. A., Fahlgren, M. K., \& McCloskey, M. S. (2020). The relationship between resting heart rate variability, hostility, and in vivo aggression among young adults. Journal of Aggression, Maltreatment \& Trauma, 29(2), 206-222.

33. Puhalla, A. A., \& McCloskey, M. S. (2020). The relationship between physiological reactivity to provocation and emotion dysregulation with proactive and reactive aggression. Biological psychology, 155, 107931.

34. Roos, L. E., Knight, E. L., Beauchamp, K. G., Berkman, E. T., Faraday, K., Hyslop, K., \& Fisher, P. A. (2017). Acute stress impairs inhibitory control based on individual differences in parasympathetic nervous system activity. Biological psychology, 125, 58-63.

35. Sassenrath, C., Barthelmäs, M., Saur, J., \& Keller, J. (2021). Inducing empathy affects cardiovascular reactivity reflected in changes in high-frequency heart rate variability. Cognition and emotion, 35(2), 393-399.

36. Shaffer, F., \& Ginsberg, J. (2017). An overview of heart rate variability metrics and norms. Frontiers in public health, 5, 258.

37. Sloan, R. P., Shapiro, P. A., Bigger Jr, J. T., Bagiella, E., Steinman, R. C., \& Gorman, J. M. (1994). Cardiac autonomic control and hostility in healthy subjects. The American journal of cardiology, 74(3), 298-300.

38. Thayer, J. F., Hansen, A. L., Saus-Rose, E., \& Johnsen, B. H. (2009). Heart rate variability, prefrontal neural function, and cognitive performance: the neurovisceral integration perspective on selfregulation, adaptation, and health. Annals of Behavioral Medicine, 37(2), 141-153.

39. Thomson, N., Gillespie, S., \& Centifanti, L. (2020). Callous-unemotional traits and fearlessness: A cardiovascular psychophysiological perspective in two adolescent samples using virtual reality. Development and Psychopathology, 32(3), 803-815.

40. Tottenham, N., Tanaka, J. W., Leon, A. C., McCarry, T., Nurse, M., Hare, T. A., . . Nelson, C. (2009). The NimStim set of facial expressions: judgments from untrained research participants. Psychiatry research, 168(3), 242-249. 
41. Tracy, L. M., \& Giummarra, M. J. (2017). Sex differences in empathy for pain: What is the role of autonomic regulation? Psychophysiology, 54(10), 1549-1558.

42. Umhau, J. C., George, D. T., Reed, S., Petrulis, S. G., Rawlings, R., \& Porges, S. W. (2002). Atypical autonomic regulation in perpetrators of violent domestic abuse. Psychophysiology, 39(2), 117-123.

43. Williams, E. D., Steptoe, A., Chambers, J. C., \& Kooner, J. S. (2011). Ethnic and gender differences in the relationship between hostility and metabolic and autonomic risk factors for coronary heart disease. Psychosomatic Medicine, 73(1), 53-58.

44. Zygmunt, A., \& Stanczyk, J. J. A. o. m. s. A. (2010). Methods of evaluation of autonomic nervous system function. $6(1), 11$.

\section{Figures}

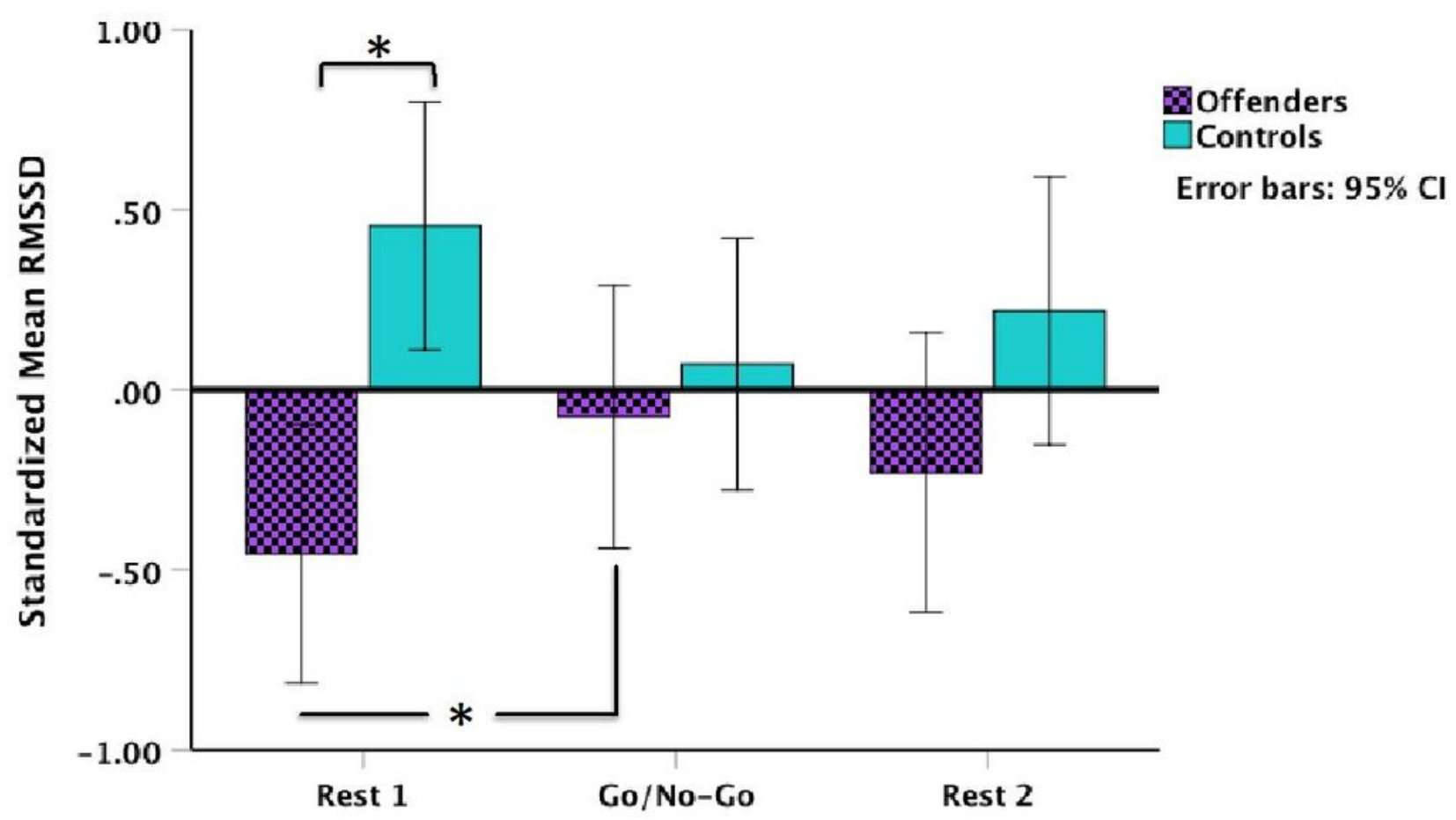

Figure 1

Parasympathetic activity (standardized RMSSD values) in the offender and control groups at baseline (Rest 1), during the emotional Go/No-Go task, and at recovery (Rest 2). Offenders' RMSSD mean was significantly lower than the controls at baseline, but increased significantly during the task and to a similar level as the controls. ${ }^{*} p<0.05$. 